\title{
The dynamic turn in quantum logic
}

\author{
Alexandru Baltag • Sonja Smets
}

Received: 7 February 2011 / Revised: 16 February 2011 / Accepted: 19 February 2011 /

Published online: 7 June 2011

(C) The Author(s) 2011. This article is published with open access at Springerlink.com

\begin{abstract}
In this paper we show how ideas coming from two areas of research in logic can reinforce each other. The first such line of inquiry concerns the "dynamic turn" in logic and especially the formalisms inspired by Propositional Dynamic Logic (PDL); while the second line concerns research into the logical foundations of Quantum Physics, and in particular the area known as Operational Quantum Logic, as developed by Jauch and Piron (Helve Phys Acta 42:842-848, 1969), Piron (Foundations of Quantum Physics, 1976). By bringing these areas together we explain the basic ingredients of Dynamic Quantum Logic, a new direction of research in the logical foundations of physics.
\end{abstract}

Keywords Dynamic quantum logic · Logical dynamics ·

Operational quantum logic · Logical foundations of physics ·

Quantum information

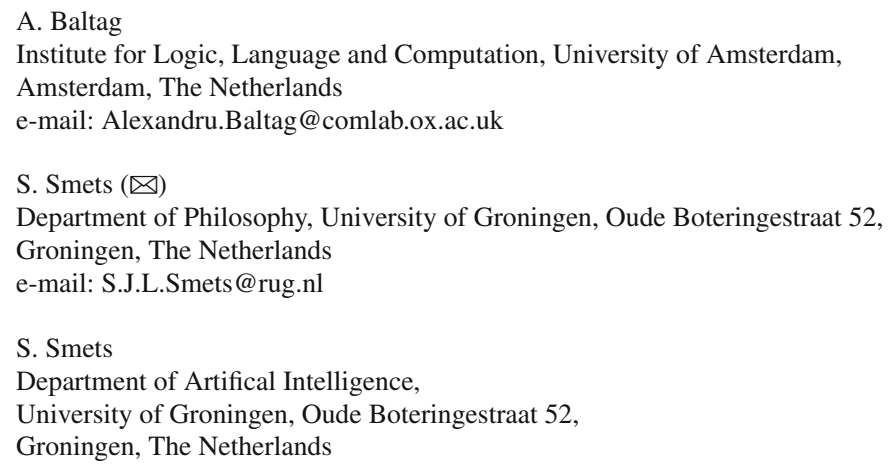




\section{Introduction}

The work of von Neumann in (1932) and the paper he co-authored with Birkhoff in (1936) are generally considered to form the birth of Quantum Logic. In their seminal paper, Birkhoff and von Neumann argue that the logic underlying the formalism of Quantum Mechanics is not classical logic. They show that the logical structure of "experimental propositions" describing the testable properties of quantum systems is non-Boolean, unlike the corresponding structure in the case of "classical mechanical" physical systems. A few decades after its publication, this paper fueled a famous debate among philosophers and logicians about whether or not Quantum Mechanics requires giving up basic principles of classical propositional logic, such as Distributivity (of Conjunction over Disjunction, and vice-versa) or even the principle of Bivalence. Surprisingly enough, the stance that has dominated the debate for most of the time was the one arguing against classical logical principles. In particular Putnam (1968) took Birkhoff and von Neumann's approach on Quantum Logic as the main motivating example in support of his claim that some of the "necessary truths" of logic can turn out to be false for empirical reasons. However, we believe that the recent results and new ideas obtained in the development of a Dynamic Approach to Quantum Logic (Baltag and Smets 2005, 2004, 2006, 2008, 2010a,b) can be used to reopen the discussion about the empirical nature of logic.

From our semantical perspective, the key distinction lies between the logical laws governing static information and the laws governing the dynamics of information. The "experimental" or "testable" properties of a quantum system are not the only meaningful propositions pertaining such systems. The logic of all such meaningful (static) quantum propositions forms a classical Boolean structure. Admittedly, the more restricted class of testable quantum properties does not form such a Boolean structure. But, in our view, the underlying reason for this failure is not that they are properties of quantum systems, but that they are dynamic properties of quantum systems. A "testable" property is one that can be tested: it is the non-classical nature of quantum tests that explains their different logical behavior. A test is an information-extracting action, and so it is the non-classical dynamics of quantum information that makes all the difference. Our conclusion is that a correct logical understanding of quantum phenomena doesn't require any revision of the classical (Boolean) logic of "static" propositions; but it does require a non-classical logical dynamics.

The formal tools we used for this were developed as part of the recent trend towards a "dynamification" of logic (Benthem 1996; van Benthem 2010). The central idea is to look at various logical systems as codifying the formal laws governing various types of actions of information production and information processing. Logical deduction and its inference steps are specific such actions, forming the traditional building blocks in logic; but besides them there are other informational actions, such as observation, communication and computation. In the context of physics, the basic informationextracting action can be taken to be the measurement of an observable property of a physical system (represented in the traditional Hilbert-space formalism of Quantum Mechanics by using idempotent linear operators called projectors). In our work, we show that traditional quantum logic can be interpreted as a dynamic logic of quantum measurements. The specific feature of quantum systems is that observations typically 
change the observed system: there is in general no informational change without ontic change. This leads us to looking at physical systems as "dynamic-logical systems", whose state is characterized by the potential changes they can undergo. In addition to measurements, one can then consider another basic type of physical changes, namely unobserved "evolutions" of a quantum system (traditionally represented as unitary operators in the Hilbert-space formalism). Our Quantum Dynamic Logic can be understood as the logic of quantum measurements and quantum evolutions.

In this paper we do not present any new technical results, but give an overview of Quantum Dynamic Logic, putting it in its historical and philosophical context. This approach originates in the convergence of ideas from two independent research areas: first, the work on logical foundations of Quantum Mechanics, and in particular the Geneva Approach to Operational Quantum Logic, pioneered in the nineteen sixties and seventies by Jauch and Piron; the second, independent line of work is the already mentioned "dynamic turn" in logic, pursued (mainly but not exclusively) by the Dutch school in modal logic lead by van Benthem. In this paper we show how a cross-fertilization between these two lines of inquiry has lead to new ideas and results in quantum logic and we argue that this brings about a new perspective in the discussion on the empirical nature of logic. Several ingredients of this cross-fertilization will play an important role. The first is the idea of Operationalism as adopted by the Geneva Approach and how this relates to the dynamic turn in Logic. The Geneva approach in Operational Quantum Logic, incorporates features in its mathematical framework which belong to the realm of "actions and physical dynamics". An extension of these ideas into a (categorical) logic framework were later on developed in Brussels in (Amira and Stubbe 1998; Coecke et al. 2004, 2001; Coecke and Smets 2004; Coecke and Stubbe 1999; Smets 2006). On the other hand, modal logicians use "dynamics" in a context of modeling processes. The standard setting in modal logic makes use of (multi-modal) Kripke models (known in Computer Science as labeled transition systems), in which the basic ingredients are labeled binary relations on a set of states. These relations represent actions that lead from input states to output states, and are particularly useful to model computational processes in Computer Science. By combining the Geneva approach and the modal logic approach, we obtain Quantum Dynamic Logic. The power of this cross-fertilization comes from the fact that the tools used to model classical computational processes can be adapted to model quantum information flow. All that is needed is to give a quantum (re)interpretation of the language of Propositional Dynamic Logic. The "actions" in this modal logic are interpreted as quantum-physical actions, i.e. as quantum measurements and unitary evolutions. This setting is powerful in the sense that it incorporates the ideas of the Geneva school in a modal logic setting, and moreover it allows us to capture (and even go beyond) all the expressive power of traditional quantum logic.

In Sect. 2 we explain the traditional take on quantum logic which traces back to the work of Birkhoff and von Neumann. In Sect. 3 we present the operational ideas of the Geneva approach to Quantum Logic. In Sect. 4 we explain the "dynamic turn" in logic, which paves the way to Sect. 5 on "the dynamic turn in quantum logic". We end in our Conclusion with a note about how this construction sheds new light on the debate about the empirical nature of Logic. 


\section{Background: traditional quantum logic}

\subsection{Origin of quantum logic}

The origin of quantum logic traces back to a short paragraph in von Neumann's book the Grundlagen der Quantenmechanik (von Neumann 1932). It is here that von Neumann introduced the idea of a logical calculus of physical properties. He argued that it is the relation between these physical properties and the projection operators definable on a Hilbert space that should make it possible to obtain some sort of logical calculus. The details of this calculus were worked out later in the paper that von Neumann co-authored with Birkhoff in (1936). Together their aim was to discover the logical structure of quantum mechanics. According to them, the logic of quantumexperimental propositions is different from classical logic but nonetheless "resembles the usual calculus of propositions with respect to and, or and not" (Birkhoff and von Neumann 1936). The difference does not concern properties of negation (as for instance in intuitionistic logic), but rather the properties linking conjunction and disjunction. More specifically, the Distributivity law (of conjunction over disjunction, and vice-versa) is the "weakest link in the algebra of logic", according to Birkhoff and von Neumann. Hence, the distributive law is replaced by them with a weaker version, the so-called Modular Law. Later research by Husimi (1937) showed that this law should actually be further weakened, ${ }^{1}$ obtaining the so-called Orthomodular Law. ${ }^{2}$

Birkhoff and von Neumann's paper gave rise to a long tradition of research into these type of quantum structures, and in particular into the topic of Ortho-Modular Lattices (OML). Hence it's not surprising that in most of the older work, "quantum logic" as a whole has been identified with "the theory of OML's".

\subsection{Orthomodular lattices}

To explain in more detail how OML's are used to capture the structure of the experimental properties of a quantum system, let us first recall that a Hilbert space $H$ is a complex vector space with an inner product $\langle\cdot, \cdot\rangle$ which is complete in the induced metric.

States. We represent the state of any given physical system by a "ray", i.e. a onedimensional subspace

$$
s=\{\lambda \cdot x: \lambda \in C\}
$$

in a Hilbert space $H$. (Here, $\lambda \in C$ denotes an arbitrary complex scalar and $x \in H \backslash\{0\}$ is a given vector in $H$.) We denote by $\Sigma$ the state space, given by the set of all "states" (rays) of a Hilbert space $H$.

Properties. An example of a physical property would be "the observable $X$ takes value $Y$ ", where $X$ can be the position of the quantum system, its momentum or some

\footnotetext{
1 Since Modularity does not hold on infinite-dimensional Hilbert spaces.

2 Foulis remarks in (1999) that Loomis (1955) and Maeda (1955) independently rediscovered Husimi's orthomodular law in 1955.
} 
other observable, and $Y$ an element of a given result structure. In the quantum logic literature, one most often focuses only on the so-called "experimental propositions", which we denote testable properties of a quantum system. A testable property is one that can in principle be tested by a measurement. In a Hilbert space $H$, the testable properties correspond to the closed linear subspaces of $H$. Given a subspace $M \subseteq H$, we denote the orthogonal subspace by $\sim M=\{y \in H: \forall x \in M\langle x, y\rangle=0\}$, where $\langle x, y\rangle$ is the notation for inner product. A subset $M \subseteq H$ is a closed linear subspace of $H$ if and only if it is biorthogonally closed, i.e. $\sim \sim M=M$. The family of all closed linear subspaces of $H$ is denoted by $\mathcal{L}(H)$. We use the notation $\mathcal{L}$ for the family of all subsets $P=\{s \in \Sigma: s \subseteq W\}$ of $\Sigma$, corresponding to the closed linear subspaces $W \subseteq H$. Hence $\mathcal{L}$ represents the family of all testable properties on a more abstract level.

Lattice Structure. The closed linear subspaces in $\mathcal{L}(H)$ form a lattice with respect to set-theoretical inclusion $\subseteq$. Hence, in the abstract algebraic approach of traditional Quantum Logic, the family $\mathcal{L}$ of testable properties is assumed to be a lattice. The lattice-theoretic meet (greatest lower bound) of two linear subspaces is given by their intersection $M \cap N$, while their join (least upper bound) can be defined as the orthodual of the meet: $M \sqcup N:=\sim(\sim M \cap \sim N)$. In fact, the join $M \sqcup N$ of two closed linear subspaces is the closed linear subspace generated by their union $M \cup N$. Physically, the join $M \sqcup N$ can be interpreted as denoting the property of (either satisfying property $M$, or satisfying property $N$, or) being in a superposition of (states satisfying) $M$ and (states satisfying) $N$. The smallest (bottom) element of the lattice is denoted by 0 and the largest (top) element by 1 .

Orthocomplementation. The family of closed linear subspaces $\mathcal{L}(H)$ comes equipped with the map $\sim: \mathcal{L}(H) \rightarrow \mathcal{L}(H), M \mapsto \sim M$, which induces a map $\sim: \mathcal{L} \rightarrow \mathcal{L}$ on testable properties called the orthocomplementation and satisfies the following conditions for every $P, Q \in \mathcal{L}$ :

$$
\begin{aligned}
P \cap \sim P & =0 \\
P \sqcup \sim P & =1 \\
P & =\sim \sim P \\
P \subseteq Q & \Rightarrow \sim Q \subseteq \sim P
\end{aligned}
$$

Orthomodular Lattice. The lattice $(\mathcal{L}, \subseteq, \cap, \sqcup, \sim, 0,1)$ is said to be an orthomodular lattice if in addition it satisfies the Orthomodular Law (also known as the Weak Modular Law):

$$
\text { For all } P, Q \in \mathcal{L}: \quad \text { if } P \subseteq Q \text { then } Q=P \sqcup(Q \cap \sim P) \text {. }
$$

Orthomodularity is a weaker condition than distributivity of $\sqcup$ over $\cap$ (i.e. $P \sqcup(Q \cap$ $R)=(P \sqcup Q) \cap(P \sqcup R)$ and $P \cap(Q \sqcup R)=(P \cap Q) \sqcup(P \cap R))$. As stressed in Birkhoff and von Neumann (1936): "Distributivity is a law in classical, not quantum mechanics". The Orthomodular Law is satisfied by the lattice of closed linear subspaces of any Hilbert space (while the usual Distributivity of meet over join is not satisfied). 
Orthomodular Lattice versus Boolean Algebra. What the above shows is that the structure of the testable properties of a physical system is given by an OML $(\mathcal{L}, \subseteq, \cap, \sqcup, \sim, 0,1)$ with $\mathcal{L} \subseteq \mathcal{P}(\Sigma)$. Obviously, if instead we consider the logical structure of all subsets of a given state space $\Sigma$ ordered by inclusion, we would obtain a Boolean algebra. The difference is due to the fact (already mentioned above) that not all subsets of the state space correspond to testable properties.

Representation Theorem. One of the important results in this line of work on OML, concerns a representation theorem with respect to the Hilbert-Space model. The theorem (based on results by Piron (1964,1976) Solèr (1995) and Mayet (1998)) states that any orthomodular lattice satisfying a set of additional lattice-theoretic axioms can be embedded in the lattice of closed linear subspaces $\mathcal{L}(H)$, for some infinite dimensional Hilbert space $H$. For more information about this setting, we refer to Kalmbach (1983); Dalla Chiara et al. (2004); Wilce (2002).

\section{Operational quantum logic}

Originally the focus of quantum logic was to look for an axiomatic system directly based on an underlying Hilbert space structure, very much in line with the traditional work of Birkhoff and von Neumann. However, from the 1950s onwards we see a slight shift in focus. Rather than taking the Hilbert space model of quantum physics for granted, the aim was now to justify its use based on a list of more basic, independently motivated empirical or "operational" conditions and axioms. Two of the most influential lines of research in this direction were developed in the 1960s and 1970s. The first is the "Amherst approach" which goes back to the work of Foulis and Randall (1972,1973), and the second is the "Geneva approach" originating in the work of Jauch and Piron (1969); Piron (1976).

Operationalism in Philosophy of Science. The type of "operationalism" one finds in these approaches is rather different from the original "operational doctrine" of Bridgman. The work of Bridgman, a Nobel prize winner in Physics in 1946 who also wrote on the Philosophy of Science, gave rise to a theory of meaning under the motto that "we do not know the meaning of a concept unless we have a method to measure it" (Chang 2009). While this theory was influential among pragmatists and positivists for some time, it is now generally considered as an "extreme and outmoded position" in Philosophy of Science (Chang 2009). In contrast, the "operationalism" of the Amherst and Geneva approaches simply aims to characterize a physical system only by means of its potential interactions with its "environment", i.e. in terms of possible "tests" or other operations that an "observer" can perform on the system. The Amherst approach restricts itself to the "environment's perspective", and hence adopts a stance that is probably closer to a form of constructive empiricism, in which "empirical adequacy" leads the way. On the other hand, the Geneva approach adopts the "system's perspective", within a sophisticated philosophical framework of "operational realism". In the rest of this section we will mainly focus on the details of the Geneva approach, and refer the reader to Wilce (2009) for an overview and state of the art of the Amherst school in Quantum Logic.

The Geneva approach proposes a theory to characterize physical systems (ranging from classical to quantum) by means of their physical properties. These properties 
are taken to be "real" physical qualities, which have an actual extension in the real world.

Operationalism in the Geneva Approach. The "operationalism" of the Geneva approach should thus be seen as a pragmatic attitude rather than as a "pure", anti-realist doctrine in philosophy of science. The underlying assumption is that a physical system should in principle be completely describable (i.e. uniquely identifiable) in terms of its "experimental properties"; i.e. properties that are associated to explicit experimental procedures, for which we have defined in advance what would be a "positive" response (that establishes the property) should we perform the experiment. In the work of Piron, these experimental procedures are called "(yes-no) questions", while more recently they were called definite experimental projects (DEP) (Moore 1999). We can think of these procedures as forming a list of concrete actions ("questions"), to be performed on a given system, together with conditions defining a "positive" outcome of such actions. Given this list, we can assign the response "yes" to the question if the conditions are satisfied and the response "no" otherwise. The questions will form the basis for the construction of a "property lattice" in the Geneva approach.

True Questions. First, a question (or rather, the property tested by the question) is said to be true, or the corresponding DEP is said to be certain, for a particular system "if it is sure that the positive response would obtain should we perform the experiment." (Moore 1999, p. 66). In the earlier work of Piron, the definition of a true question encompassed a preparation procedure of the physical system. "When the physical system has been prepared in such a way that the physicist may affirm that in the event of an experiment the result will be "yes", we shall say that the question is certain, or that the question is true." (Piron 1976, p. 20).

Impossible Questions. A question (DEP) is said to be impossible for a particular system "if it is sure that the positive response would not obtain should we perform the experiment." (Moore 1999, p. 66). "Impossible" means here that it is certain that we would obtain the negative response "no", should we perform the experiment (see also Aerts 1983).

Not True Questions. Another important notion, introduced in Aerts (1983), is that of a not true question (not certain DEP) - to be distinguished from the previously mentioned case of impossible questions. A given definite experimental project is "not certain" (a question is "not true") for a particular system if it is not sure that the positive response would obtain should we perform the experiment. In other words, the word "not" in the expression "not true question" is simply classical negation. In contrast, the word "impossible" in the expression "impossible question" will correspond to the orthocomplement operation (the so-called "quantum negation").

Summary. Summarizing this discussion as in Aerts (1981), we see three cases: (1) we can predict the outcome of a question to be "yes" so the question is "true"; (2) we can predict the outcome of a question to be "no", so the question is "impossible", and (3) we are not able to predict the outcome to be "yes", so the question is "not true".

Trivial and Absurd Questions. Next we define a "trivial question" as "do whatever you wish with the system and assign the response "yes"." (Moore 1999, p. 67). There is also the notion of an "absurd question", given by the procedure: "do whatever you wish with the system and assign the response "no"." (Moore 1999, p. 67). The absurd question is an impossible question on any physical system: it can never be true. 
Product Questions. An important construct is given by the product question, which for a given family of questions $A$ is defined by the following procedure: "choose an $\alpha$ from the family $A$ as you wish, and effectuate it." (Moore 1999, p. 67). It is easy to see that the product question is true if, for every experiment $\alpha \in A$, it is sure that the answer "yes" would obtain should we perform the experiment $\alpha$.

Inverse Questions. The inverse question is defined by exchanging the responses "yes" and "no". Hence the absurd question is the inverse of the trivial question and vice versa. The inverse of a product question with respect to a certain family of questions, is then the product question with respect to the family of the inverse component questions. We call the inverse of a specific question, named $\alpha$, true if and only if $\alpha$ is impossible and the inverse of $\alpha$ is impossible if and only if $\alpha$ is true (see Moore 1999).

Pre-Order on Questions. For a given physical system, the collection of all yes-no questions $Q$ can be endowed in a natural way with a preorder $\prec \subseteq Q \times Q$, where $\alpha \prec \beta$ expresses that every time when question $\alpha$ is true, $\beta$ is true as well. The trivial question is the maximal element and the absurd question is the minimal element. We denote by $[\alpha]$ the equivalence class of question $\alpha$ (consisting of all questions $\beta$ for which $\alpha \prec \beta$ and $\beta \prec \alpha$ ).

Properties. We associate to each equivalence class of questions a physical ("testable") property. This is the core of the operational idea: each "physical property" is to be associated with an experimental procedure performable on a physical system.

Actual Properties. A property is said to be "actual" if and only if the questions which test it are true, and "potential" otherwise. The given preorder relation on questions then induces a partial order relation on the set of all physical properties. Within the Geneva approach, one then proves that the set of all properties of a physical system forms a complete lattice. It is the product question which operationally ensures the existence of a greatest lower bound (meet) for any subset of properties, yielding as such a complete meet semilattice (and hence, also a complete lattice).

Orthocomplemented Properties. Finally, it is physically plausible to postulate that each property is the "opposite" of another one. This allows us to introduce an orthocomplementation operation, ${ }^{3}$ i.e. a surjective map': $L \rightarrow L$ for which $a \leq b \Rightarrow$ $b^{\prime} \leq a^{\prime}, a \wedge a^{\prime}=0, a \vee a^{\prime}=1$ and $a^{\prime \prime}=a$.

Classical and Quantum Systems. Within the above setting, we can now describe both classical and quantum systems. A system is considered to be "classical" if for every property $a \in L$, there is at least one question $\alpha$ belonging to the equivalence class of questions $a$, such that for every possible state $s$ of the system, ${ }^{4}$ it is sure $a$ priori which result (yes or no) would obtain should we perform the experiment $\alpha$ on the system in state $s$. For a classical system a yes-no question or its inverse is true in every possible state of the system, while for a "pure" quantum system this is only the case for the trivial and absurd questions. For the latter kind, Piron proved in (1964)

\footnotetext{
3 Note that the definition of an orthocomplement of a physical property $a$ is rather complicated in the Geneva approach because the inverse operation on questions does not lift to an orthocomplement operation on properties. This fact has lead to many misunderstandings about the Geneva approach and was explained by Foulis and Randall in (1984).

4 In the Geneva approach the state of a physical system is defined as the collection of all its actual properties (see e.g. Piron 1972; Jauch and Piron 1969).
} 
that a property lattice which is complete, atomic, orthocomplemented and satisfies Weak Modularity and some additional conditions (the so-called Covering Law, Irreducibility and having high enough dimension) can be embedded in the lattice of closed linear subspaces of a "generalized Hilbert space". 5 Later, Solèr (1995) strengthened the hypothesis (adding more additional conditions) to prove a similar Representation with respect to the usual (infinite dimensional) Hilbert spaces (with scalars in the field of complex numbers). More recently, Mayet (1998) gave an equivalent formulation, in which the required conditions are simpler to state and more intuitive.

\section{The dynamic turn in logic}

The dynamic turn in logic is based on the underlying methodological principle of "action"-based reasoning. The traditional perspective on logic is "static", being based on the idea of assigning a fixed truth value (true or false) to logical propositions. In contrast, the dynamic perspective incorporates a mechanism for changing the truth values of propositions. Below, we briefly sketch the origin of this idea in the landscape and development of logic.

\subsection{Origins of the dynamic turn in logic}

In the last century in particular, logicians came across several types of scenarios in which the traditional way of reasoning with two static truth values proved to be too limited. One way out of this problem was the old proposal of modal logic, which incorporates the idea that truth values are "local", i.e. dependent on so-called "possible worlds", or "states" of the system.

Modal Logic. In standard modal logic the language comes equipped with additional operators that can express the necessity and the possibility of a proposition to be true. The semantics of these operators explicitly uses the local dependence of truth values on different possible worlds or states of the system. Hence, modal expressions (like "necessary" or "possible") are used "to qualify the truth of a judgement" (Garson 2009).

The older work on modal logic and in particular the notion of possibility triggered another line of work in the 1920's, namely the idea of multiplying the truth values beyond the traditional two (truth and false).

Multi-valued Logic. One of the important contributors to multi-valued logic, Jan Lukasiewicz, was indeed initially guided by the idea of understanding the notion of possibility in a 3-valued setting (Gottwald 2009). This gave rise to the idea of replacing the two fixed truth values with more and more options ranging, from three to infinitely many values, and even to truth value gaps or gluts.

Semantic Games. Another independent line of work emerged in which researchers looked at truth values as being grounded in game-theoretic concepts. Departing from the late 1950's with Leon Henkin and after that the work of Jaakko Hintikka (for an

\footnotetext{
5 A generalized Hilbert space is defined just like a Hilbert space, except that the scalars can range over the fields of real numbers, complex numbers or quaternions.
} 
overview see e.g. Hodges 2009), this field developed further into what we now know as "semantic games" or "evaluation games" in logic. The main idea is that truth corresponds to the existence of a winning strategy for the verifier of the proposition in question. As we see below, the game-theoretic definition of truth already incorporates a "dynamic" perspective in its approach, since a specific dynamic process (the playing of game) is involved in determining whether a formula is true or false.

Modeling Actions. The dynamic perspective in logic aims to "incorporate actions as first-class citizens" (Benthem 2003), i.e. to explicitly formalize the processes that establish or change the truth values of propositions. In contrast to adding more truth values, as in the multi valued approach, the dynamic view fits better to the modal logic approach: the modal idea of "locality" (dependency of truth value on state, possible world or model) is further enriched with precisely the actions that specify how can one switch between the states, possible worlds or even between the models themselves. There are various mechanisms that can trigger such changes. Depending on the type of situations at hand, these changes can be brought about by acts of communication, computation, belief revision, learning, measurements, games etc. Formally these actions are modeled by means of dynamic operators that can express the (possible or necessary) truth of a proposition after the execution of the action (should such an action be executed).

Applications. This idea of action-based reasoning, initially introduced by computer scientists, opened the road of logic to other areas of applications and has in recent years proven to be very successful. Logic as seen from this perspective is no longer constrained to the traditional study of logical consequence, but instead it can be applied to model complicated multi-agent scenarios in artificial intelligence or in social software, or to prove the security of message-passing over complex communication networks. Overall, different logical systems have been developed that fit with this general idea. In the area of computer science we refer to Hoare logic, dynamic logic, labeled transition systems, Petri nets, process algebra, automata theory, game semantics and coalgebras; while in logic and philosophy one can mention situation semantics, action logic, dynamic epistemic logic, linear logic, arrow logic and logics for belief revision. In the following section we will mainly focus on one of these logical systems, namely Propositional Dynamic Logic (PDL).

\subsection{Propositional dynamic logic}

PDL, and its fragment the Hoare Logic (see Balbiani (2007); Harel et al. (2000)), are among the main logical formalisms used in program verification, i.e. in checking that a given (classical) program meets a required specification. This logic can be used as a language to prove or verify important properties of computer programs, such as "safety" or "correctness". Formally, PDL involves dynamic modal operators for dealing with these programs.

Syntax. The syntax of PDL consists of two levels, the first level consists of static propositional formulas and the second level consists of dynamic formulas expressing actions or programs. Starting from a given set of atomic propositions $p$ and basic actions $U$, these two levels are defined by mutual induction: 


$$
\begin{aligned}
& \varphi::=\perp|p| \neg \varphi \quad|\varphi \wedge \psi|[\pi] \varphi \\
& \pi::=U|\varphi ?| \pi \cup \pi|\pi ; \pi| \pi^{*}
\end{aligned}
$$

Explanation. We use here the standard notation $\perp$ to denote the "always false" proposition, we use $\neg$ to express the classical negation and $\wedge$ for the classical conjunction. The modal operators $[\pi]$ are labeled by complex programs and are used to build a particular type of formulas $[\pi] \varphi$ that express the weakest preconditions: so $[\pi] \varphi$ means that, if program $\pi$ would be performed on the current state of the system, then the output-state would necessarily satisfy property $\varphi$. The "test" $\varphi$ ? denotes the action of testing property $\varphi$ : in the way it is defined in the semantics for PDL, this action is successful if and only if $\varphi$ is true and the state of the system is left unchanged by testing $\varphi$, in all other cases the test fails. Hence a successful test $\varphi$ ? indicates that property $\varphi$ was true before the test, and also stays true after the test. At the lower action-level we see several program constructors: the first is the operation of taking the arbitrary choice between actions $\pi \cup \pi^{\prime}$ which stands for "do either action $\pi$ or action $\pi^{\prime \prime}$. Next there is the construct of sequential composition $\pi ; \pi^{\prime}$ which stands for "first perform $\pi$ and then $\pi^{\prime \prime}$ ". The $*$ expresses iteration, hence $\pi^{*}$ stands for "repeat $\pi$ a finite number of times".

From an information theoretic perspective, PDL can be used to reason about the flow of information in a complex computer program. The program constructors are particularly well fit to express more complicated operations in classical programming such as "if then else" or "do while"-loops (Harel et al. 2000). Hence from this perspective it is natural to try to extend this framework to reason about quantum programs and quantum information flow. This will be the point of focus in the next section.

\section{Dynamic quantum logic}

We argue here that the above-mentioned methodological principle of "action"-based reasoning underlying the dynamic turn in Logic ties in nicely with the older work on the logical foundational of Quantum Mechanics, and in particular with the operational perspective of the Geneva approach. One can combine the operational perspective with a dynamic view to obtain a solid basis for a formal semantics to model the behavior of quantum systems. For the following overview we explicitly follow the presentation in Baltag and Smets (2005, 2004, 2006, 2008, 2010a); Smets (2011).

\subsection{From questions to tests}

Let us start by going back to the setting of the Geneva approach (see Sect. 3) in which each physical property of a quantum system is associated to a collection of equivalent experimental procedures (yes-no questions). In Hilbert space terms these properties correspond to the closed linear subspaces of a Hilbert space while the (yes-no) questions corresponds to a pair of projectors: one operator projects onto the given linear subspace $P$, while the other operator projects onto its orthogonal complement $\sim \mathrm{P}$. Hence Piron's questions represent binary measurements in the sense that if the answer 
"yes" is obtained, then we know that property $P$ is established: it is certain that $P$ will hold after the measurement (at the output-state); while if the answer is "no", then $\sim P$ will hold after the measurement (at the output-state) (Baltag and Smets 2010a).

Quantum Tests. Each of these two ingredients (the above two projectors) of a (yes-no) question, will now be viewed as a separate "logical action". The action corresponding to the first projector (matching to the "yes" answer) will be denoted as $P$ ?, and called the "(successful) test of property $P$ ", using the language of PDL. The action corresponding to the second projector (matching to the negative result) will be denoted by $(\sim \mathrm{P})$ ? and called the "failed (unsuccessful) test of P", or equivalently, the "( successful) test of $\sim \mathrm{P}$ ".

Extracting Information by Testing. From the result of performing a successful test $P$ ? one can only deduce that $P$ holds after the test, but this doesn't typically tell us anything about whether $P$ was true or not before the test. The classical view in which measurements are used to "discover" information about an unchanged reality is no longer applicable to the situation at hand. All procedures for extracting information about a quantum system are such that the very act of observing typically changes the original system under observation. Hence the only thing we know is that the successfully tested property became true, while at the same time the original state might have changed: a successful test can be said to "actualize" the tested property. We do know a bit more, namely that the tested property became stably true: the immediate repetition of the same test will certainly give the same result. (In Hilbert space terms this corresponds to the fact that the orthogonal projectors are idempotent).

Quantum Incompatibility and the Non-monotonicity of Quantum Learning. We will view the successful quantum tests of a given proposition as a form of information-update. However as indicated above, there is a clear difference between the impact of quantum and classical forms of information update. In the quantum case the successful tests have an ontic impact on the state of the system, which does not occur in the classical case. As a consequence, the information obtained by a successful test can be overwritten by performing further tests: so the results of quantum tests do not usually cumulate in a monotonic manner! This occurs when we consider different consecutive tests of properties referring to so-called "incompatible" or complementary observables (such as for instance position and momentum): the successful test of one of the observables nullifies the results of any previous test of the other observable. Hence the notion of quantum incompatibility or "complementarity" arises naturally from the semantics of quantum tests.

\subsection{Quantum transition systems}

At the basis of the dynamic turn in quantum logic lies the development of a quantum version of PDL (Baltag and Smets 2005, 2004, 2006, 2008, 2010a; Smets 2011). As we saw before, standard PDL involves dynamic modal operators for dealing with actions or programs. One of the PDL actions was the so-called "test" of a proposition. In standard PDL, this is an action that is possible only when the corresponding proposition holds (on the given system), in which case the state of the system remains the same after the action. In a quantum version of PDL, the semantics of testing of a 
proposition will have to be different, namely it will be given by the above-defined "quantum test" actions. This formalism was later extended to cover other forms of quantum "actions", such as the quantum "evolutions" (quantum-logical gates), represented in a Hilbert space by unitary operators. We can also close the class of our quantum-logical actions under the standard operations with non-deterministic programs (sequential composition, and arbitrary infinitary choice), obtaining a natural class of "quantum programs".

Formally, our dynamic models are non-classical relational models of Propositional Dynamic Logic, called "Quantum Transition Systems" (QTS). Any infinite-dimensional Hilbert space can be naturally structured in this way (Baltag and Smets 2005).

Dynamic Frames. First we define the concept of a dynamic frame as a labeled transition system $\mathcal{F}=\left(\Sigma,\{\stackrel{P ?}{\rightarrow}\}_{P \in \mathcal{L}},\{\stackrel{U}{\rightarrow}\}_{U \in \mathcal{U}}\right)$, consisting of:

1. a set $\Sigma$ of objects, called states;

2. a family of binary "transition" relations $\stackrel{P ?}{\rightarrow} \subseteq \Sigma \times \Sigma$, labeled by "test" actions $P$ ?; the action labels come from a given family $\mathcal{L} \subseteq \mathcal{P}(\Sigma)$ of subsets $P \subseteq \Sigma$, called testable properties;

3. a family of binary "transition" relations $\stackrel{U}{\rightarrow} \subseteq \Sigma \times \Sigma$, labeled by basic "actions" $U \in \mathcal{U}$, which we will call basic unitary evolutions.

Measurement Relation. Next we equip any dynamic frame $\mathcal{F}$ with a so-called measurement relation:

$$
s \rightarrow t \text { iff } s \stackrel{P ?}{\rightarrow} t \text { for some } P \in \mathcal{L}
$$

Orthogonality Relation. The negation of the measurement relation gives rise to what is known in the literature as an orthogonality relation:

$$
s \perp t \text { iff } s \not \rightarrow t
$$

Note that for any set $S \subseteq \Sigma$, we write $t \perp S$ iff we have $t \perp s$ for all $s \in S$. We also define the orthogonal (or orthocomplement) of the set $S$ by $\sim S:=\{t \in \Sigma: t \perp S\}$. In line with Sect. 2, the biorthogonal closure of a set $S$ is simply the set $\sim S=\sim(\sim S)$.

Special Case of Classical PDL. The Kripke frames for classical PDL can now be seen as a special case of these dynamic frames. In this case $\mathcal{L}$ is given by $\mathcal{P}(\Sigma)$ and the transition relation for a test is given by $s \stackrel{P ?}{\rightarrow} t$ iff $s=t \in P$ in which case the "measurement relation" is simply the identity $s=t$. In contrast, a quantum version of dynamic frames will be more complicated. $\Sigma$ is then given by the set of states of a quantum physical system, orthogonality will be non-trivial and the testable properties are not given by the powerset of $\Sigma$ but instead by the biorthogonally closed subsets of $\Sigma$.

Quantum Transition Systems. A QTS (or quantum dynamic frame) is a dynamic frame $\mathcal{F}=\left(\Sigma,\{\stackrel{P}{\rightarrow}\}_{P \in \mathcal{L}},\{\stackrel{U}{\rightarrow}\}_{U \in \mathcal{U}}\right)$, satisfying the following list of conditions (in which variables $P, Q$ range over testable properties in $\mathcal{L}$, variables $s, t, s^{\prime}, t^{\prime}, v, w$ range over states in $\Sigma$ and $U$ ranges over evolutions) Baltag and Smets (2005): 


\section{Frame Conditions.}

1. Closure under arbitrary conjunctions: if $\mathcal{L}^{\prime} \subseteq \mathcal{L}$ then $\bigcap \mathcal{L}^{\prime} \in \mathcal{L}$

2. Atomicity. States are testable, i.e. $\{s\} \in \mathcal{L}$.

This is equivalent to requiring that "states can be distinguished by tests", i.e. if $s \neq t$ then $\exists P \in \mathcal{L}: s \perp P, t \not \perp P$

3. Adequacy. Testing a true property does not change the state: if $s \in P$ then $s \stackrel{P ?}{\rightarrow} s$

4. Repeatability. Any property holds after it has been successfully tested: if $s \stackrel{P ?}{\rightarrow} t$ then $t \in P$

5. "Covering Law". If $s \stackrel{P ?}{\rightarrow} w \neq t \in P$, then there exists some $v \in P$ such that $t \rightarrow v \nrightarrow \rightarrow s$.

6. Self-Adjointness Axiom: if $s \stackrel{P \text { ? }}{\rightarrow} w \rightarrow t$ then there exists some element $v \in \Sigma$ such that $t \stackrel{P ?}{\rightarrow} v \rightarrow s$

7. Proper Superposition Axiom. Every two states of a quantum system can be properly superposed into a new state: $\forall s, t \in \Sigma \exists w \in \Sigma s \rightarrow w \rightarrow t$

8. Reversibility and Totality Axioms. Basic unitary evolutions are total bijective functions: $\forall t \in \Sigma \exists ! s s \stackrel{U}{\rightarrow} t$ and $\forall s \in \Sigma \exists ! t s \stackrel{U}{\rightarrow} t$

9. Orthogonality Preservation. Basic unitary evolutions preserve (non) orthogonality: Let $s, t, s^{\prime}, t^{\prime} \in \Sigma$ be such that $s \stackrel{U}{\rightarrow} s^{\prime}$ and $t \stackrel{U}{\rightarrow} t^{\prime}$.

Then: $s \rightarrow t$ iff $s^{\prime} \rightarrow t^{\prime}$.

10. Mayet's Condition: Orthogonal Fixed Points. There exists some unitary evolution $U \in \mathcal{U}$ and some property $P \in \mathcal{L}$, such that $U$ maps $P$ into a proper subset of itself; and moreover the set of fixed-point states of $U$ has dimension $\geq 2$. In other words:

$\exists U \in \mathcal{U} \exists P \in \mathcal{L} \exists t, w \in \Sigma \forall s \in \sim \sim\{t, w\}: U(P) \subseteq P, U(P) \neq P$, $t \perp w, U(s)=s$.

Note. These axioms imply that $\mathcal{L}$, with set-inclusion as partial order, forms an orthomodular lattice of infinite height satisfying all the necESsary conditions for the representation theorem of Piron, Solèr and Mayet to hold. The details of this result have been presented in Baltag and Smets (2005).

Concrete Quantum Transition Systems. A special case of QTS's are the “concrete" ones, given by an infinite-dimensional Hilbert space $H$. In a concrete QTS, the set of "states" $\Sigma$ is taken to be the family of all one-dimensional closed linear subspaces of $H$. The class of testable properties $\mathcal{L}$ is given by the family of closed linear subspaces of $H$. The relations of type $\stackrel{P ?}{\rightarrow}$ correspond to (successful) quantum tests given by the projectors onto the closed linear subspace corresponding to property $P$. The binary relations $\stackrel{U}{\rightarrow}$ correspond to unitary actions or linear maps $U$ on $H$. The set of "basic unitary actions" $\mathcal{U}$ is the family of all (maps induced on $\Sigma$ by) unitary operators on $H$.

In Baltag and Smets (2005) we prove the following "Abstract Soundness and Completeness" theorems for the Hilbert-space semantics: 
These results show how every (abstract) QTS can be canonically embedded in the concrete QTS associated to an infinite-dimensional Hilbert space.

Theorem 1 ("Soudness") Every “concrete QTS” (as defined above) is a QTS.

Theorem 2 ("Abstract Completeness") Every QTS is isomorphic to a Concrete Quantum Transition System.

Importance of these results. The above results are an extension of the older Hilbertcomplete axiomatizations of algebraic quantum logic, and in particular of the latticetheoretic Piron-Solér-Mayet Representation Theorem mentioned in Sect. 2. While that result gave a representation of the elements of the logical lattice as closed linear subspaces of a Hilbert space, our result extends this representation to the "dynamic" components of the logical structure (the PDL actions), which are now represented in terms of (unions of compositions of) projectors and unitary evolutions of the associated Hilbert space. Our axiomatization in terms of QTS can also be seen to extend the older work on completeness of ortho-logic with respect to its relational semantics (Dishkant 1972; Goldblatt 1974). This is now extended to full orthomodular quantum logic. By moving to dynamic frames, we solve one of the main problems posed by orthoframes (as shown in Goldblatt (1984)), namely that orthomodularity could not be captured by any first-order frame condition. In contrast, in a QTS, orthomodularity corresponds to a first-order frame condition, with a natural dynamic/operational interpretation. Similarly, the "Mayet condition" could only be stated in a lattice-theoretic setting using the second-order notion of lattice isomorphism (to encode the required unitary action); while in our dynamic-logic setting, unitary actions are first-class citizens, hence this condition can be stated in the basic language of PDL. In this sense, our result is also an improvement of the Piron-Solér-Mayet Representation Theorem. It shows yet another advantage of moving from a purely lattice-theoretic logical structure to a dynamic-logical one (consisting of two levels, one for static propositions and one for actions): certain key second-order concepts, that are indispensable for the completeness of the axiomatization, are "internalized" in this way as actions (and action modalities).

Generalized QTS. In later work (Baltag and Smets 2006, 2008, 2010a), we generalized the setting of QTS to allow dynamic frames of the form $\left(\Sigma,\{\stackrel{P ?}{\rightarrow}\}_{P \subseteq \Sigma},\{\stackrel{U}{\rightarrow}\}_{U \in \mathcal{U}}\right)$ in which the tests are now labeled by arbitrary subsets $P \subseteq \Sigma$ of the state space. Such a setting allows us to talk about arbitrary, possibly non-testable, properties of a physical system. The actions of the form $\{\stackrel{P ?}{\rightarrow}\}$ still represent "quantum tests": but now the property that is being tested is not necessarily the property $P$ itself, but it is the strongest testable property entailed by $P$.

Testable Closure. In a generalized QTS, we define for any property $P \subseteq \Sigma$ its testable closure $\bar{P}$ as the strongest testable property that is entailed by it, i.e. $\bar{P}=$ $\bigcap\{Q \in \mathcal{L}: P \subseteq Q\}$. In a Hilbert space, $\bar{P}$ is simply the closed linear subspace generated by the set $P$. Moreover, in a Hilbert space, the testable closure is the same as the biorthogonal closure: $\bar{P}=\sim \sim P$.

Generalized Tests. We can now associate a "generalized test" program $P$ ? to any property $P \subseteq \Sigma$, including the non-testable ones. This is defined as the test of the testable closure of $P$, i.e. $P$ ? $=\bar{P}$ ? 


\subsection{The logic of quantum actions ( $L Q A)$}

Language LQA. The language of our dynamic quantum logic can now be taken to be the star-free fragment of PDL (i.e. the Kleene star operator is eliminated) with "converse" for some of the actions (the basic evolutions). More concretely, this language consists of one level for sentences $\varphi$ (denoting "static" propositions), and another level for programs (or actions) $\pi$, levels defined by mutual recursion:

$$
\begin{aligned}
& \varphi::=p|c| \neg \varphi|\varphi \wedge \psi|[\pi] \varphi \\
& \pi::=U\left|U^{\dagger}\right| \varphi ?|\pi \cup \pi| \pi ; \pi
\end{aligned}
$$

Semantics. This language can now be interpreted in a generalized Quantum Transition System, by interpreting tests $\varphi$ ? as quantum tests (of the strongest testable property entailed by $\varphi$ ), the basic actions $U$ as quantum gates and $U^{\dagger}$ as the inverse gates (or evolutions). To interpret the propositional constants $c$, we need to enrich our generalized QTS by assigning a standard interpretation $\|c\| \subseteq \Sigma$ to each constant. Further, we keep the classical interpretation for all the other operators. In particular we keep the Boolean negation and the classical conjunction. The program expressions $\pi$ can now be interpreted as quantum programs. In a given QTS $\mathcal{F}$, the class of quantum programs over $\mathcal{F}$ is defined as the smallest family of binary relations $Q \subseteq \mathcal{P}(\Sigma \times \Sigma)$ which contains all tests $\{\stackrel{P}{\rightarrow}\}_{P \in \mathcal{L}}$, all basic gates $\{\stackrel{U}{\rightarrow}\}_{U \in \mathcal{U}}$ as well as their inverses $\stackrel{U^{-1}}{\rightarrow}$, and is closed under the operations of relational composition $R ; R$ and arbitrary union of relations $\bigcup_{i \in I} R_{i}$. The resulting logic has been called the Logic of Quantum Actions ( $L Q A)$.

LQA is a Bivalent Logic. Obviously, $L Q A$ is a bivalent, Boolean logic. All the propositional connectives satisfy all the classical laws of propositional logic. The dynamic-logic formulas denote possible properties of quantum states. The classical negation $\neg \varphi$ of a given property $\varphi$ simply expresses now the fact that property $\varphi$ does not hold. However, not all the expressible properties are "testable properties" in the sense we introduced before. For example, the classical negation of a testable property might not itself be testable. Syntactically, the testable properties can be characterized as the ones that can be expressed by negation-free formulas of $L Q A$ : any formula built without the use of (classical) negation denotes a testable property.

Abbreviations in the syntax. We keep the standard abbreviations from $P D L$ for the dual operator $\langle\pi\rangle \varphi$, bottom $\perp$ and top $\top$. We also put:

$$
\begin{aligned}
\sim \varphi & :=[\varphi ?] \perp(\text { orthocomplement }) \\
\nabla \varphi & :=\langle\varphi ?\rangle \top(\text { possibility of successful test }) \\
\square \varphi & :=[\neg \varphi ?] \perp(\text { certainty of successful test) } \\
\varphi \leq \psi & :=\square \square(\varphi \rightarrow \psi)(\text { entailment }) \\
\varphi=\psi & :=\square \square(\varphi \leftrightarrow \psi)(\text { equivalence }) \\
\bar{\varphi} & :=\sim \sim \varphi=\square \diamond \varphi \text { (testable closure) } \\
T(\varphi) & :=(\varphi=\bar{\varphi})(\text { the formula is testable) }
\end{aligned}
$$


Modal Axioms. Above we defined a QTS as a dynamic frame satisfying ten conditions. For each semantic condition, we can now give a modal axiom (in the language of $P D L)$ that "captures" it in the sense of correspondence theory in modal logic. Hence a dynamic frame makes an axiom valid if and only if it satisfies the corresponding condition. But in some cases (e.g. atomicity, proper superpositions), this is not possible and the axioms can then only be captured in a weak sense (for a weak version of the condition).

Proof System. We include all the axioms and all the inference rules of PDL (except for the axioms referring to Kleene star). In addition, we have the following:

\section{LQA Inference Rules.}

- Testable Generalization Rule.

From $\theta \rightarrow[p$ ?] $\varphi$, infer $\theta \rightarrow \square \varphi$, provided that $p$ does not occur in $\theta$ or $\varphi$.

- Atomicity Rule.

From $\theta \wedge T(p) \wedge p \leq \varphi \rightarrow p \leq \psi$, infer $\theta \rightarrow \varphi \leq \psi$, provided that $p$ does not occur in $\theta, \varphi, \psi$.

\section{LQA Axioms.}

1. Test: $\square \varphi \rightarrow[p ?] \varphi$

2. Testability of constants: $T(c)$

3. Partial Functionality: $\neg[p ?] q \rightarrow[p ?] \neg q$

4. Adequacy: $(p \wedge q) \rightarrow\langle p ?\rangle q$

5. Repeatability: $T(p) \rightarrow[p ?] p$

6. Proper Superpositions:

$\langle\pi\rangle \varphi \rightarrow\left[\pi^{\prime}\right] \nabla^{2} \varphi$

7. "Covering Law":

$T(q) \wedge p \wedge \square(p \rightarrow \diamond[p ?] q) \rightarrow q$

8. Self-Adjointeness:

$p \rightarrow[q ?] \square\langle q ?\rangle \diamond p$

9. Reversibility and Totality of Actions:

$p \leftrightarrow\left[U ; U^{\dagger}\right] p$ and $p \leftrightarrow\left[U^{\dagger} ; U\right] p$

10. Orthogonality Preservation:

$[U] \square p \leftrightarrow \square[U] p$

11. Mayet's Conditions:

$\diamond^{2} a, \diamond^{2} b, \diamond^{2} c, \diamond^{2} d$

$a \rightarrow[u](a \wedge \neg b)$

$c \rightarrow \neg \diamond d$

$(\varphi \wedge \square \vee(c \vee d)) \rightarrow[u] \varphi$.

Expressive Power of LQA. Note that the presence of a classical negation in $L Q A$ increases the expressivity of our language: $L Q A$ is strictly more expressive than traditional Quantum Logic. Using classical negation, we defined the classical dual $\langle\varphi ?\rangle \psi:=\neg[\varphi$ ?] $\neg \psi$ of the weakest precondition, which expresses the possibility of actualizing a property $\psi$ by a successful test of property $\varphi$. This also allows us to express the possibility of performing a (successful) test for $\varphi$, via $\nabla \varphi:=\langle\varphi$ ? $\rangle$ T. Moreover, LQA is strictly more expressive than traditional Quantum Logic even with respect to quantum-testable properties. Indeed, the use of classical negation allows us 
to express new testable properties: e.g. for any arbitrary property $P$, the expression $\square P=\neg \diamond \neg P$ denotes a testable property; while the expression $\square \diamond P$ captures the strongest testable property entailed by $P$.

An important result in our work Baltag and Smets (2005, 2006, 2008, 2010a) shows that traditional quantum logic can be re-interpreted inside $L Q A$. This can be achieved by defining the orthocomplement $\sim \varphi$ of a property as the impossibility of a successful test, i.e. by putting:

$$
\sim \varphi:=[\varphi ?] \perp
$$

The quantum join is definable via de (quantum) Morgan law, by putting:

$$
\varphi \sqcup \psi:=\sim(\sim \varphi \wedge \sim \psi)
$$

This yields a dynamic interpretation of all the non-classical connectives of traditional quantum logic.

Embedding of Traditional QL into LQA. This interpretation can be used to embed traditional quantum logic into $L Q A$, via a faithful translation $t r$ into $L Q A$, defined recursively:

$$
\begin{aligned}
\operatorname{tr}(p) & =\square \diamond p \\
\operatorname{tr}(\sim \varphi) & =[\operatorname{tr}(\varphi) ?] \perp \\
\operatorname{tr}(\varphi \wedge \psi) & =\operatorname{tr}(\varphi) \wedge \operatorname{tr}(\psi) .
\end{aligned}
$$

As mentioned in Baltag and Smets (2010a), this translation is faithful on Hilbert spaces: if $\Sigma(H)$ is the concrete QTS induced by $H$, then for all states $s$ and all quantum logic formulas $\varphi$ we have

$$
s \models_{H} \varphi \text { iff } s \models \Sigma(H) \operatorname{tr}(\varphi) .
$$

As a consequence of these results we obtain the following theorem:

Theorem 3 LQA is a conservative extension of traditional Quantum Logic: i.e. for all formulas $\varphi$ of traditional $Q L$, we have

$$
\models Q L \varphi \text { iff }=_{L Q A} \operatorname{tr}(\varphi)
$$

Embedding of LQA- into Traditional QL. One can also see that traditional Quantum Logic is in fact equivalent (co-expressive) on Hilbert spaces with the negation-free test-only fragment of $L Q A$, fragment denoted by $L Q A^{-}$. To make this precise, we define recursively a faithful translation $t r^{\prime}$ of the language of $L Q A^{-}$into the language of traditional quantum logic:

$$
\begin{aligned}
\operatorname{tr}^{\prime}(p) & =p \\
t r^{\prime}([\varphi ?] \psi) & =\sim t r^{\prime}(\varphi) \sqcup\left(t r^{\prime}(\varphi) \wedge t r^{\prime}(\psi)\right) \\
t r^{\prime}(\varphi \wedge \psi) & =t r^{\prime}(\varphi) \wedge t r^{\prime}(\psi)
\end{aligned}
$$


Theorem 4 Quantum Logic is the negation-free test-only fragment of $L Q A$ : every formula of LQA built without the use of (classical) negation or of unitary actions $U$ is equivalent to a formula of traditional Quantum Logic (and vice-versa). More precisely, for all formulas $\varphi$ of $L Q A^{-}$, we have

$$
\models_{L Q A^{-}} \varphi \quad \text { iff } \models Q L \operatorname{tr}^{\prime}(\varphi)
$$

Notes. We have shown that traditional quantum logic can be embedded into a classical propositional dynamic logic $L Q A$ equipped with special quantum actions. The fact that $L Q A$ is strictly more expressive than traditional Quantum Logic is an important observation and casts new light on the logical foundations of Quantum Mechanics. In $L Q A$ we can now represent (using evolutions and classical negation) new quantum properties, including testable properties, that were not expressible in the traditional logical framework. Our results can also be cast in algebraic terms. As the work of Kozen and Pratt in the late seventies and early eighties indicates, an algebraic semantics for a PDL can be presented as a dynamic algebra of actions. In Baltag and Smets (2005) we presented a different axiomatization in terms of Quantum Dynamic Algebras, giving a complete axiomatization of the algebra of quantum actions.

\section{Conclusion}

We have shown in this paper how ideas coming from two different logical traditions, namely the operational quantum logic approach of the Geneva school and the dynamic turn in modal logic, were combined to produce our "dynamic quantum logic" setting.

From a philosophical perspective, our approach sheds new light on the old debate about the nature of quantum logic. In contrast to common opinions (see e.g. Putnam 1968), we found no empirical ground to abandon any principles of classical logic. Our logical approach can explain all the non-classical features of quantum behavior ${ }^{6}$ as a consequence of the non-classical "dynamics" of quantum information. Our setting does not require any change of the classical laws governing "static" information. It is the non-classical nature of quantum-information-extracting actions (quantum tests) that explains the strangeness of quantum behavior. If one treats the quantumlogical operators appropriately (in terms of axioms about dynamic modalities), then quantum-logical laws can safely coexist within the same logical calculus with the laws of classical propositional logic: indeed the logic $L Q A$ (see Sect. 5) provides precisely such a setting.

Finally, our logical treatment of quantum systems does justice to the one foundational principle that is common to the Geneva approach and to the Dynamic turn in logic, namely the idea that a system is completely characterized by its potential behavior under all possible interactions. This fertile idea, having both deep philosophical

\footnotetext{
6 Although the logic presented here deals only with single physical systems, the setting was extended in Baltag and Smets $(2004,2006)$ to deal with compound systems, and to explain in dynamical terms more complex quantum phenomena such as entanglement, teleportation, no cloning and the power of quantum computation.
} 
implications and wide applications in Computer Science, lies also at the core of our Quantum Dynamic Logic.

Acknowledgements We thank Johan van Benthem for his valuable comments on this paper. Sonja Smets thanks the Netherlands Organization for Scientific Research for sponsoring this work under the VIDI research programme with number 639.072.904.

Open Access This article is distributed under the terms of the Creative Commons Attribution Noncommercial License which permits any noncommercial use, distribution, and reproduction in any medium, provided the original author(s) and source are credited.

\section{References}

Aerts, D. (1981). The one and the many, towards a unification of the quantum and the classical description of one and many physical entities. PhD-thesis, Free University of Brussels.

Aerts, D. (1983). The description of one and many physical systems. In C. Gruber, C. Piron, T. M. TL'm, R. Weill (Eds.), Les fondements de la mö́canique quantique, 25e cours de perfectionnement de l'Association Vaudoise des Chercheurs en Physique Montana, du 6 au 12 mars 1983 (pp. 63-148). Lausanne: 1'A.V.C.P.

Amira, H. B., \& Stubbe, I. (1998). How quantales emerge by introducing induction within the operational approach. Helvetica Physica Acta, 71, 554-572.

Balbiani, P. (2007) Propositional Dynamic Logic. Online entry of the Stanford Encyclopedia of Philosophy

Baltag, A., \& Smets, S. (2005). Complete axiomatizations for quantum actions. International Journal of Theoretical Physics, 44(12), 2267-2282.

Baltag, A., \& Smets, S. (2004). The Logic of Quantum Programs. In The proceedings of the 2nd international workshop on quantum programming languages (QPL 2004), TUCS general publication no. 33, Turku Center for Computer Science.

Baltag, A., \& Smets, S. (2006). LQP: The dynamic logic of quantum information. Mathematical Structures in Computer Science, Special Issue on Quantum Programming Languages, 16(3), 491-525.

Baltag, A., \& Smets S. (2008). A dynamic-logical perspective on quantum behavior. In I. Douven, \& L. Horsten (Eds.), Special issue: Applied logic in the philosophy of science. Studia logica, 89, 185-209.

Baltag, A., \& Smets, S. (2010a). Quantum logic as a dynamic logic. In T. Kuipers, J. van Benthem, \& H. Visser (Eds.), Synthese, 179(2), 285-306.

Baltag, A., \& Smets, S. (2010b). Correlated knowledge, an epistemic-logic view on quantum entanglement. International Journal of Theoretical Physics, 49(12), 3005-3021.

Birkhoff, G., \& von Neumann, J. (1936). The logic of quantum mechanics. Annals of Mathematics, 37, 823-843. Reprinted in C. A. Hooker (Ed.). (1975). The logico-algebraic approach to quantum mechanics (Vol. 1, pp. 1-26). Dordrecht: D. Reidel Publishing Company.

Chang, H. (2009). Operationalism. In Stanford encyclopedia of philosophy.

Coecke, B., Moore, D. J., \& Smets, S. (2004). Logic of dynamics \& dynamics of logic; some paradigm examples. In S. Rahman, J. Symons, D. M. Gabbay \& J. P. Van Bendegem (Eds.), Logic, epistemology and the unity of science (pp. 527-556). Dordrecht: Kluwer.

Coecke, B., Moore, D. J., \& Stubbe, I. (2001). Quantaloids describing causation and propagation for physical properties. Foundations of Physics Letters, 14, 357-367.

Coecke, B., \& Smets, S. (2004). The Sasaki Hook is not a [static] implicative connective but induces a backward [in time] dynamic one that assigns causes. International Journal of Theoretical Physics, 43, 1705-1736.

Coecke, B., \& Stubbe, I. (1999). On a duality of quantales emerging from an operational resolution. International Journal of Theoretical Physics, 38, 3269-3281.

Dalla Chiara, M., Giuntini, R., \& Greechie, R. (2004). Reasoning in quantum theory. Dordrecht: Kluwer Academic Pub.

Dishkant, H. (1972). Semantics of the minimal logic of quantum mechanics. Studia Logica, 30, 23-30.

Foulis, D. (1999). A half century of quantum logic ? What have we learned? In: D. Aerts \& J. Pykacz (Eds.), Quantum structures and the nature of reality (pp. 1-36). Dordrecht: Kluwer Acad. Pub. 
Foulis, D. J., \& Randall, C. H. (1972). Operational statistics I., basic concepts. Journal of Mathematical Physics, 13, 1667-1675.

Foulis, D. J., \& Randall, C. H. (1984). A note on misunderstandings of piron's axioms for quantum mechanics. Foundations of Physics, 14, 65-81.

Garson, J. (2009). "Modal Logic", online entry of the Stanford Encyclopedia of Philosophy.

Goldblatt, R. (1974). Semantic analysis of orthologic. Journal of Philosophical Logic, 3, 19-35.

Goldblatt, R. (1984). Orthomodularity is not elementary. The Journal of Symbolic Logic, 49, 401-404.

Gottwald, S. (2009). Many-valued logic. In Online entry of the Stanford encyclopedia of philosophy.

Harel, D., Kozen, D., \& Tiuryn, J. (2000). Dynamic logic. Cambridge: MIT Press.

Hodges, W. (2009). Logic and games. In Online entry of the Stanford encyclopedia of philosophy

Husimi, K. (1937). Studies on the foundations of quantum mechanics I. Proceedings of PhysicoMathematical Society Japan, 9, 766-778.

Jauch, J. M., \& Piron, C. (1969). On the structure of quantal proposition systems. Helvetica Physica Acta, 42, 842-848.

Kalmbach, G. (1983). Orthomodular lattices. NY: Academic Press.

Loomis, L. (1955). The lattice theoretic background of the dimension theory of operator algebras. Memoirs of the American Mathematical Society No. 18.

Maeda, S. (1955). Dimension functions on certain general lattices. Journal of Science of the Hiroshima University A, 19, 211-237.

Mayet, R. (1998). Some characterizations of the underlying division ring of a Hilbert lattice by automorphisms. International Journal of Theoretical Physics, 37(1), 109-114.

Moore, D. J. (1999). On state spaces and property lattices. Studies in History and Philosophy of Modern Physics, 30, 61-83.

Piron, C. (1964). Axiomatique quantique (PhD-Thesis). Helvetica Physica Acta, 37, 439-468. Quantum axiomatics (M. Cole, Trans.). RB4 Technical memo 107/106/104, GPO Engineering Department, London.

Piron, C. (1972). Survey of general quantum physics. Foundations of physics, 2, 287-314. Reprinted in C. A. Hooker (Ed.). (1975). The logico-algebraic approach to quantum mechanics (Vol. I). Dordrecht: D. Reidel Publishing Company.

Piron, C. (1976). Foundations of quantum physics. Massachusetts: W.A. Benjamin Inc.

Putnam, H. (1968). Is logic empirical?. In R. Cohen \& M. Wartofsky, Boston studies in the philosophy of science (Vol. 5). Holland, Dordrecht: D. Reidel.

Randall, C. H., \& Foulis, D. J. (1973). Operational statistics II, manuals of operations and their logics. Journal of Mathematical Physics, 14, 1472-1480.

Smets, S. (2006). From intuitionistic logic to dynamic operational quantum logic. Poznan Studies in Philosophy and the Humanities, 91, 257-275.

Smets, S. (2011). Logic and quantum physics, Journal of the Indian Council of Philosophical Research.

Solèr, M. P. (1995). Characterization of Hilbert spaces by orthomodular spaces. Communications in Algebra, 23(1), 219-243.

van Benthem, J. (1996). Exploring logical dynamics, studies in logic, language and information. Stanford: CSLI Publications.

van Benthem, J. (2003). A mini-guide to logic in action. Research Reports PP-2004-02, University of Amsterdam

van Benthem, J. (2010). Dynamics of information and interaction. New York: Cambridge University Press.

von Neumann, J. (1932). Grundlagen der Quantenmechanik. Berlin: Springer Verlag (English translation: Mathematical foundations of quantum mechanics. New Jersey: Princeton University Press, 1996).

Wilce, A. (2002). Quantum logic and probability theory. In Stanford encyclopedia of philosophy.

Wilce, A. (2009). Test spaces. In K. Engesser, D. Gabbay, \& D. Lehmann (Eds.), Handbook of quantum logic and quantum structures (pp. 443-550). North-Holland: Elsevier 